\title{
The Thatcher illusion: Rotating the viewer instead of the picture
}

Janek S Lobmaier

Department of Psychology, University of Zurich, Ramistrasse 71, CH 8006 Zurich, Switzerland

Fred W Mast 9

Institut de Psychologie, Université de Lausanne, Bâtiment Anthropole, CH 1015 Lausanne, Switzerland; e-mail: fred.mast@unil.ch

Received 7 September 2005, in revised form 8 March 2006; published online 14 March 2007

\begin{abstract}
Faces are difficult to recognise when presented upside down. This effect of face inversion was effectively demonstrated with the 'Thatcher illusion' by Thompson (1980 Perception 9 483-484). It has been tacitly assumed that this effect is due to inversion relative to retinal coordinates. Here we tested whether it is due to egocentric (ie retinal) inversion or whether the orientation of the body with respect to gravity also influences the face-inversion effect. A 3-D human turntable was used to test subjects in 5 different body-tilt (roll) orientations: $0^{\circ}, 45^{\circ}, 90^{\circ}, 135^{\circ}$, and $180^{\circ}$. The stimuli consisted of 4 'normal' and 4 'thatcherised' faces and were presented in 8 different orientations in the picture plane. The subjects had to decide in a yes - no task whether the faces were 'normal' or 'thatcherised'. Analysis of the $d^{\prime}$ 'values revealed a significant effect of stimulus orientation and body tilt. The significant effect of body tilt was due to a drop in $d^{\prime}$ values in the $135^{\circ}$ orientation. This result is compared to findings of studies on the subjective visual vertical, where larger errors occurred in body-tilt orientations between $90^{\circ}$ and $180^{\circ}$. The present findings suggest that the face-inversion effect relies mainly on retinal coordinates, but that in head-down body-tilt orientations around $135^{\circ}$ the gravitational reference frame has a major influence on the perception of faces.
\end{abstract}

\section{Introduction}

Visual objects are difficult to recognise when presented upside down. The effect of inversion differs depending on the type of visual stimuli, faces showing a significantly larger inversion effect than objects (Yin 1969; for an overview see Valentine 1988). A widely accepted explanation for this discrepancy goes back to the distinction between featural and configural information (eg Leder and Bruce 2000). Featural information refers to information that is contained in the local parts (eg the individual shape of the nose); configural information refers to the spatial arrangement of the parts (eg the distance between the eyes and the mouth). In faces, configural information plays a dominant role, while object recognition is much more based on local information contained in the features (Biederman 1987; Marr 1982; Tversky and Hemenway 1984). Configural information has been shown to be more orientationsensitive than featural information (eg Leder et al 2001; Nachson and Sechory 2002; Searcy and Bartlett 1996), and therefore face recognition is hampered when faces are presented upside down. In this orientation, faces can only be recognised by matching their parts (Rock 1973). Thompson (1980) effectively demonstrated that different orientation sensitivities of features and configurations may be responsible for the face inversion effect (FIE). He took a photograph of the former British prime minister and inverted eyes and mouth with respect to the whole face. Such a face looks extremely grotesque when viewed right-side up, but loses this grotesqueness when the face is inverted. This effect is now commonly referred to as the Thatcher illusion. A possible explanation for this effect has been provided by Rock (1973). Recognition of such an inverted 'thatcherised' face requires featural and configural information to be rotated mentally.

- Author to whom all correspondence should be addressed. 
Yet, the spatial transformation of all features and configurations overtaxes the capacity of the underlying mechanism (cf Rock 1973). Therefore it is difficult to mentally visualise what an inverted thatcherised face would look like right-side up. The closer the orientation to upright the better the configural information can be extracted from the face. Numerous studies have been concerned with this phenomenon since then (Lewis 2001; Rakover 1999; Stürzel and Spillmann 2000; Valentine and Bruce 1985). Stürzel and Spillmann (2000) gradually turned different thatcherised faces from $0^{\circ}$ through $180^{\circ}$ and asked participants to report when the face switched from pleasant to grotesque, or vice versa. They found a relatively narrow changeover zone, between $97.2^{\circ}$ and $118.3^{\circ}$, where the change of expression occurred. Stürzel and Spillmann suggested that the striking change may be based on the step-tuning properties of hypothetical face neurons, rather than a gradual tuning curve. According to Stürzel and Spillmann, face neurons respond best to faces in a tuning width of $\pm 100^{\circ}$ relative to the vertical. They claim that these face neurons may also respond to inverted faces, but inappropriately. In contrast, Lewis (2001) reported a gradual loss of configural information the further a face is turned away from upright. He recorded the reaction times of forty participants while they discriminated thatcherised from normal faces which were presented in 10 different orientations. These two studies leave, however, the nature of the dependence on the rotation angle still equivocal.

Interestingly, what almost all studies of the FIE have in common is that they were conducted with upright observers. Therefore, it is often tacitly assumed that the effects induced by inverted stimuli are defined with respect to retinal coordinates. However, a stimulus can be upright or inverted either with respect to retinal coordinates or with respect to gravitational coordinates. Even though we are upright most of the time, it is not evident that the reference frame underlying the FIE is of purely retinal origin. It is possible that it also depends on the orientation of the face stimulus with respect to the direction of gravity, since influence of extraretinal information has been found in object recognition (Simons et al 2002). In everyday life, we mainly see faces in a gravitationally upright orientation, so it is plausible to assume that the direction of gravity can be implicitly encoded when faces are learned. In the upright body orientation, however, we are unable to disentangle the role of gravitational and retinal information because the two frames of reference are fully aligned. To investigate the influence of the gravitational frame of reference one must therefore test participants not only in the upright body orientation but also when they are tilted.

The fact that body tilt can influence visual perception has already been demonstrated by Rock (1973). A square tilted $45^{\circ}$ was no longer perceived as a square, but as a diamond. However, when the subject was tilted $45^{\circ}$ and the square remained upright (resulting in roughly the same retinal image) subjects reported that they saw a square. Gaunet and Berthoz (2000) investigated the effect of gravity on the recognition of spatial environment. Their participants were tested upright and tilted $33^{\circ}$ to the left and right. The task was to recognise photographed scenes, which were tilted in $15^{\circ}$ steps from $0^{\circ}$ to $90^{\circ}$. In contrast to Rock (1973), they found that gravity was only slightly important for recognising scenes, and concluded that in their task it played no crucial role. Recent work by Clément and Eckardt (2005) suggested that visual illusions such as the Ponzo illusion occurred less frequently when participants were lying on their side or supine compared to upright. In contrast, a study by Prinzmetal and Beck (2001) showed that the effect of visual illusions was in fact increased when observers were tilted $30^{\circ}$. Lipshits and McIntyre (1999) suggested a multisensory reference frame for the internal representation of visual stimuli. They showed a sequence of two lines of equal length which differed in orientation. The task was to memorise the orientation of the first line (reference line) and rotate the second line to the same orientation as the reference line with a rotary knob. In the upright body 
orientation, there was a clear advantage for reference lines that were horizontal and vertical (oblique effect). However, this preference disappeared when the lines were presented retinally horizontal and vertical, but the participants were tilted $22.5^{\circ}$ to the left or right. When the same task was performed under microgravity conditions $(0 g)$, the same preference as in upright body orientation was found (Lipshits et al 2005). These findings suggest that visual stimuli may be stored in a multimodal frame of reference that includes information about gravity, but that in the absence of gravity the retinal reference frame suffices to determine the oblique effect. Buchanan-Smith and Heeley (1993) provided further evidence that the oblique effect cannot simply be explained by the retinal reference frame.

Moreover, tasks involving mental image transformations have also been shown to depend on body orientation. Corballis et al (1978) tested participants in upright orientation and when they were tilted $60^{\circ}$ and $90^{\circ}$ to the side, and found that in a mental-rotation task with alphanumeric and letter-like symbols as stimuli the gravitational reference frame indeed had an influence. Specifically, they found that 'upright' is more aligned with the gravitational vertical than with the retinal vertical. Mast et al (2003) investigated the performance in four different mental imagery tasks while participants were upright, horizontal, or supine. They found an influence of body orientation in two imagery tasks, suggesting that body tilt influences at least some processes associated with mental imagery.

Does body orientation have an influence on face recognition? On the basis of the findings reported above it can be hypothesised that the direction of gravity may also have an influence on the perception of faces. In particular, the visual illusions used in the studies by Prinzmetal and Beck (2001) and Clément and Eckardt (2005) are indeed with visual stimuli involving predominantly configural processing. Since the FIE is based widely on the processing of configural information, it is possible that it is also affected by body tilt. To our knowledge, only one study has so far been conducted of the FIE in different body-tilt orientations. Troje (2003) reported no changes depending on body orientation and concluded that the retinal frame of reference is responsible for the FIE. However, the subjects in this study were upright or lying $90^{\circ}$ on the side. Therefore, it seems premature to draw conclusions that are based on only one bodytilt orientation. It is now important to study the FIE over a wider range of body tilt angles. In the present study we made use of the Thatcher illusion to investigate the FIE. We investigated whether body tilt influences the Thatcher illusion, or whether this illusion can be fully explained by stimulus orientation with respect to retinal coordinates. Using different body tilts, we disentangled the gravitational and retinal frames of reference. If, indeed, the gravitational reference frame influences the Thatcher illusion, we would expect a differential effect of body tilt on stimulus orientation. If the Thatcher illusion is based only on retinal coordinates no effect of body tilt would be expected.

\section{Experiment}

\subsection{Method}

2.1.1 Participants. Thirteen participants aged between 25 and 34 years voluntarily took part in this experiment. All but two participants reported to be right-handed. They could choose whether they wanted to be paid for participation or to receive course credits. All had normal or corrected-to-normal vision. Informed consent for participation was given prior to the experiment and the study was approved according to the Declaration of Helsinki (1991).

2.1.2 Stimuli. Four faces provided by the Max Planck Institute for Biological Cybernetics in Tübingen, Germany served as stimuli. The thatcherised stimuli were prepared with Adobe Photoshop ${ }^{\odot}$. With the elliptic tool, the eyes and mouth were cut out with a 


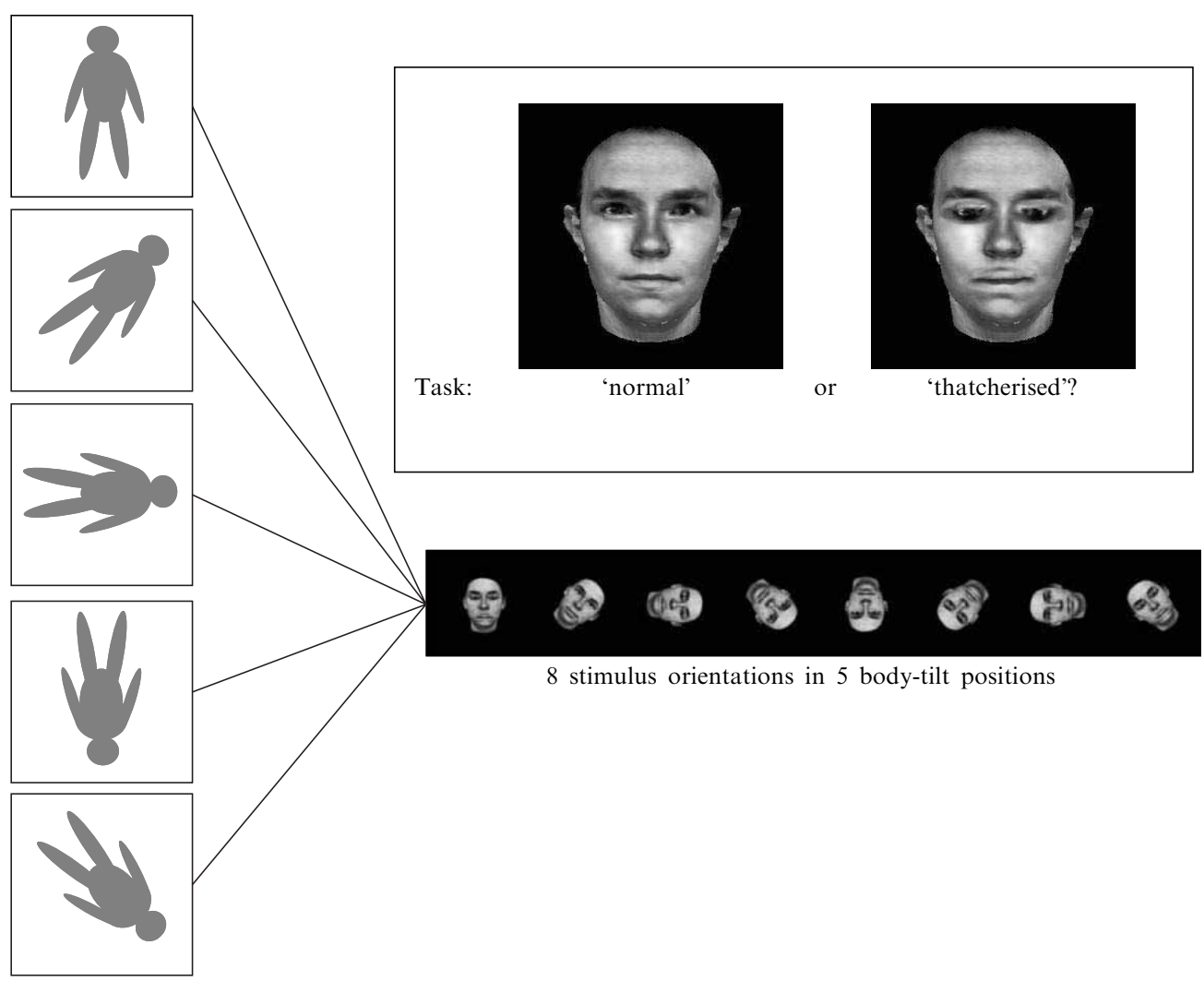

Figure 1. Design and sample stimulus: In each of 5 body tilt orientations 4 thatcherised and normal faces were presented in 8 stimulus orientations.

soft-contour feather of 5 pixels and were mirror-reversed round the horizontal axis. A sample stimulus can be seen in figure 1. Each face was then rotated in the picture plane by 8 different angles $\left(0^{\circ}, 45^{\circ}, 90^{\circ}, 135^{\circ}, 180^{\circ}, 225^{\circ}, 270^{\circ}\right.$, and $\left.315^{\circ}\right)$.

In a pilot study, a separate group of nine participants was tested outside the turntable in upright body orientation with the upright $\left(0^{\circ}\right)$ and inverted $\left(180^{\circ}\right)$ stimuli described above. The effect of stimulus orientation on accuracy was significant $\left(F_{1,4}=9.765\right.$, MSE $=4.925, p<0.05)$, showing that inverted thatcherised faces were not detected as accurately as upright thatcherised faces. The result of this pre-test demonstrated that the stimuli were appropriate for testing the FIE.

2.1.3 Apparatus. The participants were tested in 5 different body tilt orientations (roll), $0^{\circ}$ (upright), $45^{\circ}, 90^{\circ}$ (horizontal, right ear down), $135^{\circ}, 180^{\circ}$ (upside down). The face stimuli appeared in 8 different orientations $\left(0^{\circ}, 45^{\circ}, 90^{\circ}, 135^{\circ}, 180^{\circ}, 225^{\circ}, 270^{\circ}\right.$, and $315^{\circ}$ ). Thus, $8 \times 5$ combinations were possible per stimulus. 4 different individual faces were used which appeared in a thatcherised and a normal version. In total, 320 different trials were applied $(8 \times 5 \times 8)$. The experiment was run with a 3 -D human turntable (Acutronik, Jona, Switzerland) at the Department of Neurology, University Hospital, Zurich. The turntable consisted of three servo-controlled motor-driven axes which could be separately controlled.

The participants were seated on a chair mounted on the 3-D human turntable and firmly secured with safety belts. Participant's naso-occipital axis was aligned with the centre of rotation. We restrained the head with a thermoplastic mask (Sinmed BV, Reeuwijk, The Netherlands), which was individually moulded for each participant. 
The mask was attached to the back of the chair ensuring effective restraint of the head without discomfort. This fixation, in combination with the belts, ensured a stable position in head-down body tilts. The participants were brought to one of the 4 bodytilt orientations $\left(45^{\circ}, 90^{\circ}, 135^{\circ}\right.$ or $\left.180^{\circ}\right)$ with a speed of $45^{\circ} \mathrm{s}^{-1}$ and an acceleration of $45^{\circ} \mathrm{s}^{-2}$. After a delay of $2 \mathrm{~s}$ the participants were prompted to start the first trial by pressing one of the response buttons. Jaggi-Schwarz and Hess (2003; personal communication with Hess) found no torsional nystagmus (VOR) $2 \mathrm{~s}$ after stopping the body rotation at this speed. Therefore, this interval is long enough for vestibular-driven eye movements to dissipate, which could interfere with the perceptual encoding of the face stimuli. The stimuli were presented through a Macintosh G3 Powerbook which was mounted on a frame attached to the chair, with the use of PsyScope software (Cohen et al 1993). The participants responded by using a PsyScope button box which was attached to the frame. Reaction times and button presses were recorded.

2.1.4 Design. A within-subjects design was used. Participants completed two blocks of 32 trials in each body orientation, encompassing all stimulus orientations and faces, but each face appeared either normal or thatcherised. Whether a face was thatcherised or normal was pseudo-randomised with the constraint that half of the stimulus orientations of each face were thatcherised and half were normal. Thatcherisation was counterbalanced within-subjects between blocks. The order of body-tilt orientations was as follows: 4 random orders of the 5 body orientations were created; by using Latin squares, 5 orders were generated from each random order. Thus, 20 orders were computed. Each participant underwent 2 orders of body orientations. The order of the trials in each block was randomised online.

2.1.5 Task and procedure. The participants were given written and oral instructions. The task was to decide whether a face was 'normal' or 'thatcherised' by pressing the corresponding key on the response box. The participants were tilted into one of 5 body orientations and were then presented with the first test face. Each test face was presented for $200 \mathrm{~ms}$ in one of 8 stimulus orientations, either normal or thatcherised, and the participants had to respond as fast and as accurately as possible. After each block, the participants were brought back to the upright body orientation and were able to take a rest. The length of the break was self-paced, but the minimum duration was $30 \mathrm{~s}$. As soon as the participants were ready, they were tilted into the next body orientation and the experiment continued with the next block.

\subsection{Analysis}

$d^{\prime}$ values and reaction times (RTs) of the correct responses were analysed. Less than $0.8 \%$ of the trials were treated as outliers and were excluded from analysis because RTs were above $3000 \mathrm{~ms}$. $d^{\prime}$ values were calculated for each subject by subtracting the $z$-transformed false alarm rate from the $z$-transformed hit rate. First, $5 \times 8$ analyses of variance (ANOVAs) were run including all body-tilt orientations and all stimulus orientations. In addition, to investigate the FIE, $5 \times 2$ ANOVAs were conducted with all five body tilts and upright and inverted face stimuli as within-subjects factors.

\subsection{Results}

Mean $d^{\prime}$ values for each body-tilt orientation and stimulus orientation are illustrated in figure 2. Figure $2 \mathrm{a}$ shows the $d^{\prime}$ values of all stimulus orientations, independent of body tilt; figure $2 \mathrm{~b}$ shows the effect of body tilt on $d^{\prime}$ for upright and inverted stimuli. The $5 \times 8$ ANOVA on the $d^{\prime}$ values revealed a significant effect of body tilt $\left(F_{4,48}=6.307, \mathrm{MSE}=1.346, p<0.001\right)$ and of stimulus orientation $\left(F_{7,84}=39.481\right.$, MSE $=3.285, p<0.001)$, and a significant body-tilt $\times$ stimulus-orientation interaction $\left(F_{28,336}=1.751, \mathrm{MSE}=2.238, p<0.05\right)$. To investigate specifically the FIE, we computed a $5 \times 2$ ANOVA on the $d^{\prime}$ values of retinally upright and retinally inverted stimuli 


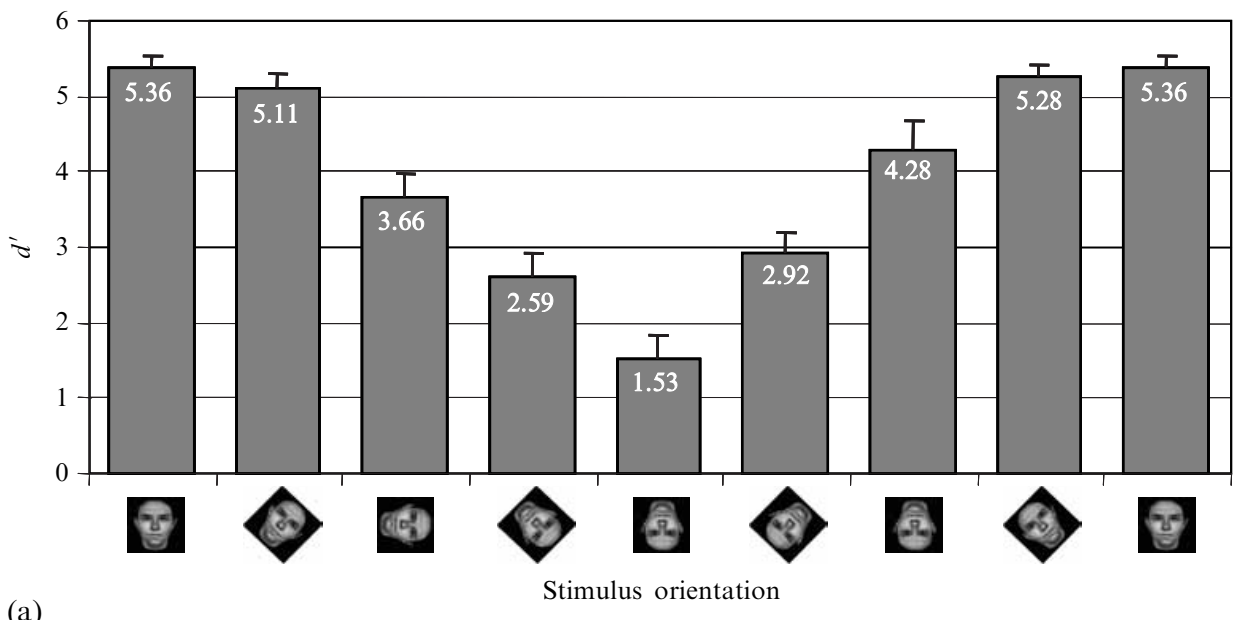

(a)

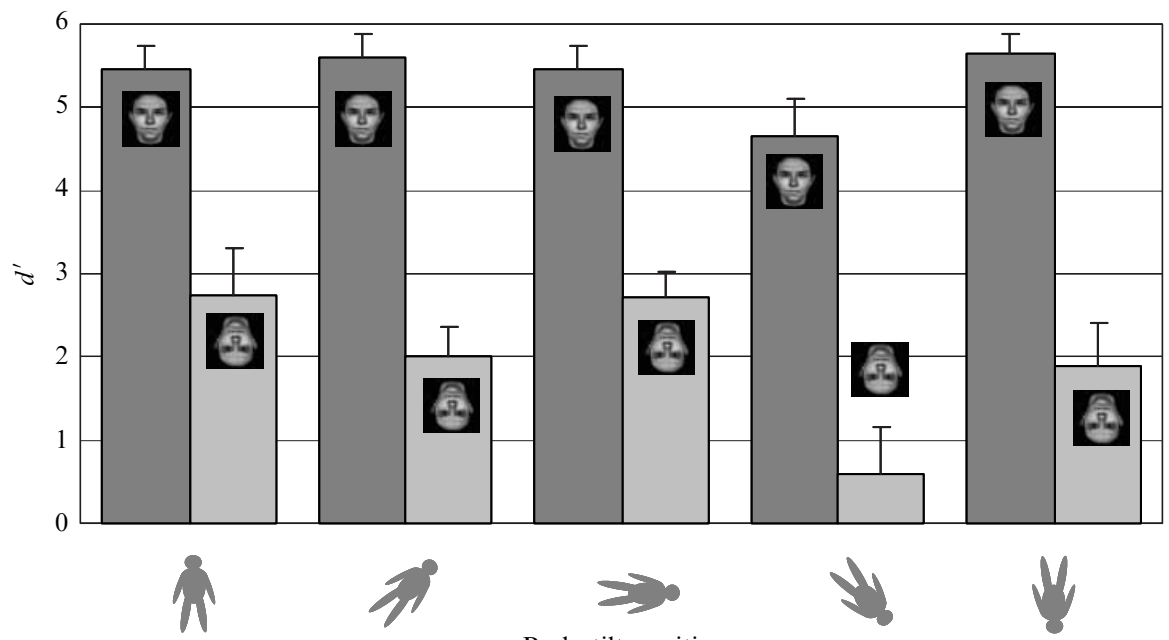

(b)

Body-tilt position

Figure 2. (a) Main effect of stimulus orientation. Mean $d^{\prime}$ values of all stimulus orientations independent of body tilt. Error bars depict standard errors of the mean, \pm 1 SEM. (b) Main effect of body tilt. Mean $d^{\prime}$ values of all body tilt orientations for retinally upright and inverted stimuli. Error bars depict \pm 1 SEM.

in all 5 body tilts, which revealed significant main effects of body tilt $\left(F_{4,48}=6.24\right.$, $\mathrm{MSE}=1.56, \quad p<0.001)$ and stimulus orientation $\left(F_{1,12}=85.10, \quad \mathrm{MSE}=4.33\right.$, $p<0.001)$. The interaction (body tilt $\times$ stimulus orientation) did not reach statistical significance $(p=0.222)$. A posteriori pairwise comparisons (Bonferroni corrected) revealed that the main effect of body tilt was due to the tilt angle of $135^{\circ}$. Here, participants had lower $d^{\prime}$ values. Only comparisons involving the $135^{\circ}$ orientation reached statistical significance: the comparison between $0^{\circ}$ and $135^{\circ}$ revealed a significant difference $(p<0.05)$, and so did the comparison between $90^{\circ}$ and $135^{\circ}(p<0.01)$. The comparison between $45^{\circ}$ and $135^{\circ}$ reached marginal significance $(p=0.11)$, and the comparison between $180^{\circ}$ and $135^{\circ}$ did not reach statistical significance $(p=0.304)$.

The mean RTs are shown in figure 3. Figure 3a illustrates the RTs of all stimulus orientations, independent of body tilt and figure $3 \mathrm{~b}$ shows the effect of body tilt on the RTs for retinally upright and inverted stimuli. The $5 \times 8$ ANOVA on the RTs revealed a main effect of stimulus orientation $\left(F_{7,84}=16.709, \operatorname{MSE}=29277, p<0.001\right)$, 


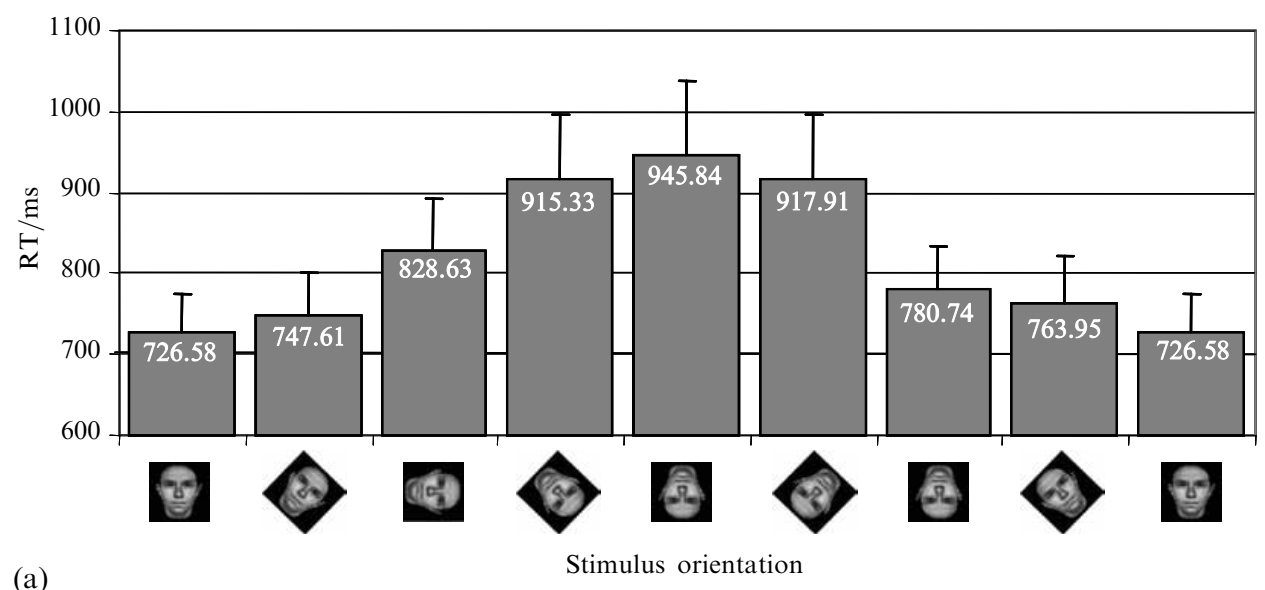

(a)

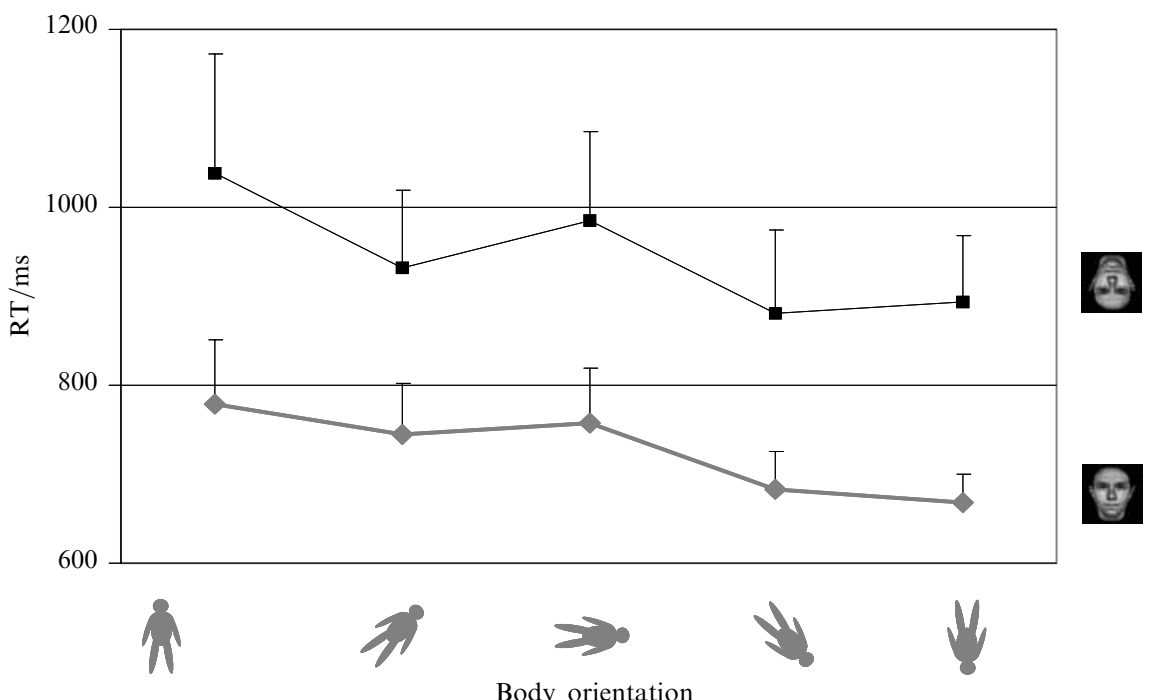

(b)

Body orientation

Figure 3. (a) Main effect of stimulus orientation. Mean RTs of all stimulus orientations independent of body tilt. Error bars depict standard errors of the mean, \pm 1 SEM. (b) Main effect of body tilt. Mean RTs of all body tilt orientations for retinally upright and inverted stimuli. Error bars depict \pm 1 SEM.

but neither the main effect of body tilt nor the interaction body tilt $\times$ stimulus orientation reached statistical significance.

The $5 \times 2$ ANOVA revealed a main effect of body tilt $\left(F_{4,48}=3.6, \mathrm{MSE}=22528\right.$, $p<0.05)$. The more participants were turned away from upright, the faster they responded. It is worth noting that $d^{\prime}$ values did not decrease in the upside-down body orientation, and therefore the shorter RTs were not due to a speed-accuracy tradeoff. The effect of stimulus orientation also reached statistical significance $\left(F_{1,12}=18.19\right.$, MSE $=85879, p<0.01$ ), but the interaction (body-tilt $\times$ stimulus-orientation) was not significant $(p=0.69)$.

\section{Discussion}

Three findings of this study deserve special attention. First, the inversion effect was generally based on retinal coordinates. Second, when the body was tilted $135^{\circ}$, thatcherised faces were more difficult to detect. Third, the further observers were 
tilted away from upright the faster they detected retinally inverted and retinally upright thatcherised faces.

Faces were more difficult to process when they were retinally inverted than when they were retinally upright. In fact, it took participants more time to discriminate thatcherised from normal faces the more they were rotated away from retinal upright. This finding is in line with the results of Lewis (2001) who reported a gradual increase of RTs with increasing stimulus orientation of thatcherised faces. Whereas Lewis (2001) reported only an increase of RTs, we also found a decrease of $d^{\prime}$. This finding indicates that configural information is gradually hampered the further a face is turned away from retinal upright. Our data therefore contradict the findings of Stürzel and Spillmann (2000), who reported a relatively narrow range of stimulus rotation angle where a thatcherised face loses its grotesqueness. They suggested that step-tuning properties of face neurons may be responsible for their results. The increasing RTs for rotated faces make it more likely to suggest a mental rotation process that underlies the findings from this study. Observers had to mentally rotate each face to a retinal upright orientation, and the time to perform this process increased with angle of stimulus orientation; at the same time the accuracy decreased.

Yet another important finding is that the angle of body tilt had an influence on the detection of the Thatcher illusion. In particular, at $135^{\circ}$ it was more difficult to detect the changes in the faces. This finding suggests that gravitational direction indeed has an influence on the FIE. It is noteworthy that $d^{\prime}$ in the upside-down body orientation $\left(180^{\circ}\right)$ did not differ from any of the other body orientations, and thus the results are not explicable by a general decline in performance caused by head-down orientations. De Schonen et al (1998) studied the FIE in microgravity and found no change when compared to performance on the ground. In microgravity, however, there is no sensory information regarding the direction of gravity and participants rely exclusively on visual information. Similarly, Troje (2003) reported no effect of body orientation on the FIE. However, his study was confined to two different body-tilt orientations only. When looking only at $0^{\circ}$ and $90^{\circ}$, our data confirm the findings of Troje (2003). Including a wider range of body-tilt orientations revealed that the direction of gravity can influence the FIE. Gaunet and Berthoz (2000) tested the influence of gravity in a naturalscene recognition task. They also used only one small body tilt $\left(33^{\circ}\right)$, and concluded that gravity is not a crucial factor in their experiment. Our present findings suggest that an effect of gravity may indeed have been found in body tilts around $135^{\circ}$. For natural scenes the effect of gravity may even exceed the effect we found for faces, because-unlike faces - natural scenes always appear gravitationally upright.

What could be the reason for the distinctive decline in performance at $135^{\circ}$ ? In this context it is interesting to note that studies of the subjective visual vertical report largest errors in body-tilt orientations about $135^{\circ}$ (eg Kaptein and Van Gisbergen 2004; Schöne 1964; Udo de Haes 1970; Van Beuzekom and Van Gisbergen 2000). Interestingly, not only the deviation from the physical vertical, but also the variance of the subjective visual vertical reached its maximum in head-down body tilts between $120^{\circ}$ and $150^{\circ}$ (Mast 2000; Mittelstaedt 1999). This indicates that participants have less reliable reference information for the perception of the vertical. As a consequence, the participants have difficulties in judging the orientation of visual stimuli with respect to gravity. The retinal and gravitational references are not aligned in $45^{\circ}, 90^{\circ}$, and $135^{\circ}$; but in $135^{\circ}$ the deviation between the retinal up and the perceived gravitational up is largest. Here, the two references deviate by more than $90^{\circ}$ (this is also true for the upsidedown orientation but there the reference frames are again aligned, albeit in exactly opposite directions). This disparity may result in a reduced confidence in the spatial reference information underlying the FIE and thus finally disrupts task performance. 
We assume that the FIE can be explained through retinal coordinates as long as the retinal and gravitational frames of reference do not deviate substantially (by more than $90^{\circ}$ ). No such conflict arises in the upside-down orientation where the reference frames are again perfectly aligned but point in opposite directions. Therefore, the FIE is essentially the result of visual information processing and only in headdown orientations around $135^{\circ}$ the extraretinal information unfolds its effect. In the present study, however, we only tested roll orientations. It will have to be the aim of future studies to investigate whether rotations round other body axes produce a similar effect (eg head-down tilts in the body pitch direction). The overall interaction of body tilt and stimulus orientation further suggests that body tilt has an influence on the perception of orientation-sensitive stimuli such as thatcherised faces. Thus, we were able to demonstrate in this study that a gravity-based component exists and can interfere with task performance.

Finally, it seems that 'standing on one's head' speeds up detection of retinally upright and inverted thatcherised faces. Taking the $d^{\prime}$ values into account it is unlikely that participants just wanted to get over more quickly with the head-down body conditions, as the detection ability of thatcherised faces did not decrease in body-tilt orientations of $180^{\circ}$. Furthermore, this advantage of being upside down applied only to retinally upright and inverted faces. Whether this finding was specific to the task used in this study and why it only occurred for upright and inverted faces will have to be the subject of further studies. As the participants in this study stayed in one body-tilt orientation for approximately $60 \mathrm{~s}$ maximally, nothing can be said about the time course for longer time periods. This enhancement may eventually decline after a certain time, presumably when the cardio-vascular system has regularised the blood pressure.

In conclusion, our study has shown that the Thatcher illusion is based mainly on the orientation of the face stimulus with respect to the retinal reference frame. However, in head-down body-tilt orientations about $135^{\circ}$ the gravitational and retinal reference frames deviate substantially and as a consequence participants have difficulties in unambiguously perceiving the orientation of visual stimuli. We found this effect for faces, whether it also applies for other complex visual stimuli will have to be the subject of future studies.

Acknowledgments. This research was supported by a grant from the Swiss National Science Foundation (project No. 611-066052) awarded to the second author, and by the Betty \& David Koetser Foundation for Brain Research. We thank Walter Schmid, Henri Gossweiler, Erika Lorincz, and Albert Züger for technical support, and Bernhard Hess for expert advice.

\section{References}

Biederman I, 1987 "Recognition-by-components: A theory of human image understanding" Psychological Review $94115-147$

Buchanan-Smith H M, Heeley D W, 1993 "Anisotropic axes in orientation perception are not retinotopically mapped" Perception 221389 - 1402

Clément G, Eckardt J, 2005 "Influence of the gravitational vertical on geometric visual illusions" Acta Astronautica $56911-917$

Cohen J D, MacWhinney B, Flatt M, Provost J, 1993 "PsyScope: A new graphic interactive environment for designing psychology experiments" Behavioral Research Methods, Instruments, and Computers $25257-271$

Corballis M C, Nagourney B A, Shetzer L I, Stefanatos G, 1978 "Mental rotation under head tilt: factors influencing the location of the subjective reference frame" Perception \& Psychophysics $24263-273$

De Schonen S, Leone G, Lipshits M, 1998 "The face inversion effect in microgravity: is gravity used as a spatial reference for complex object recognition?" Acta Astronautica 42 287-301

Gaunet F, Berthoz A, 2000 "Mental rotation for spatial environment recognition" Cognitive Brain Research 9 91-102

Jaggi-Schwarz K, Hess B J M, 2003 "Influence of dynamic tilts on the perception of earth-vertical" Experimental Brain Research $149340-350$ 
Kaptein R G, Van Gisbergen J A, 2004 "Interpretation of a discontinuity in the sense of verticality at large body tilt" Journal of Neurophysiology $912205-2214$

Leder H, Bruce V, 2000 "When inverted faces are recognized: the role of configural information in face recognition" Quarterly Journal of Experimental Psychology 53513 - 536

Leder H, Candrian G, Huber O, Bruce V, 2001 "Configural features in the context of upright and inverted faces" Perception $3073-83$

Lewis M B, 2001 “The Lady's not for turning: Rotation of the Thatcher illusion” Perception 30 $769-774$

Lipshits M, Bengoetxea A, Cheron G, McIntyre J, 2005 "Two reference frames for visual perception in two gravity conditions" Perception 34 545-555

Lipshits M, McIntyre J, 1999 "Gravity affects the preferred vertical and horizontal in visual perception of orientation" NeuroReport $101085-1089$

Marr D, 1982 Vision (San Francisco, CA: W H Freeman)

Mast F W, 2000 "Does the world rock when the eyes roll? Allocentric orientation representation, ocular counterroll, and the subjective visual vertical" Swiss Journal of Psychology 59 89-101

Mast F W, Ganis G, Christie S, Kosslyn S M, 2003 "Four types of visual mental imagery processing in upright and tilted observers" Cognitive Brain Research $17238-247$

Mittelstaedt H, 1999 "The role of the otoliths in perception of the vertical and path integration", in Otolith Function in Spatial Orientation and Movement Eds B Cohen, B J M Hess (New York: New York Academy of Sciences) pp 334-344

Nachson I, Sechory M, 2002 "Effect of inversion on the recognition of external and internal facial features" Acta Psychologica $109227-238$

Prinzmetal W, Beck D M, 2001 "The tilt-constancy theory of visual illusions" Journal of Experimental Psychology: Human Perception and Performance $27206-217$

Rakover S S, 1999 “Thompson's Margaret Thatcher illusion: when inversion fails” Perception 28 $1227-1230$

Rock I, 1973 Orientation and Form (New York: Academic Press)

Schöne H, 1964 "On the role of gravity in human spatial orientation" Aerospace Medicine 35 $764-772$

Searcy J H, Bartlett J C, 1996 "Inversion and processing of component and spatial-relational information of faces" Journal of Experimental Psychology: Human Perception and Performance $22904-915$

Simons D J, Wang R F, Roddenberry D, 2002 "Object recognition is mediated by extraretinal information" Perception \& Psychophysics $64521-530$

Stürzel F, Spillmann L, 2000 "Thatcher illusion: Dependence on angle of rotation" Perception $29937-942$

Thompson P, 1980 "Margaret Thatcher: A new illusion" Perception 9 483-484

Troje N F, 2003 "Reference frames for orientation anisotropies in face recognition and biologicalmotion perception" Perception 32 201-210

Tversky B, Hemenway K, 1984 “Objects, parts, and categories” Journal of Experimental Psychology: General $113169-193$

Udo de Haes H A, 1970 "Stability of apparent vertical and ocular countertorsion as a function of lateral tilt" Perception \& Psychophysics 8 137-142

Valentine T, 1988 "Upside-down faces: A review of the effects of inversion upon face recognition" British Journal of Psychology $79471-491$

Valentine T, Bruce V, 1985 "What's up? The Margaret Thatcher illusion revisited" Perception 14 $515-516$

Van Beuzekom A D, Van Gisbergen J A M, 2000 "Properties of the internal representation of gravity inferred from spatial-direction and body-tilt estimates" Journal of Neurophysiology $\mathbf{8 4}$ $11-27$

Yin R K, 1969 "Looking at upside-down faces” Journal of Experimental Psychology 81 141-145 


\section{PERTEPTION}

VOLUME 362007

www.perceptionweb.com

Conditions of use. This article may be downloaded from the Perception website for personal research by members of subscribing organisations. Authors are entitled to distribute their own article (in printed form or by e-mail) to up to 50 people. This PDF may not be placed on any website (or other online distribution system) without permission of the publisher. 